\title{
Evaluate the Utility of High-Speed Railway Opening by Forecasting the Induced Traffic with Satisfaction Investigation in China
}

\author{
Jiang $\mathrm{W}^{*}$, Yi L \\ MOE Key Laboratory for Urban Transportation Complex Systems Theory \& Technology, Beijing Jiaotong \\ University, No.3 Shangyuancun, Haidian District, Beijing 100044, P.R. China \\ Email: wujiang198908@126.com
}

\begin{abstract}
The induced traffic is newly proposed and together with the economic growth as a new indicator to measure the effectiveness of the high-speed railway opening in a more intuitive way in this study. The Matrix Completion and Canonical Correlation Analysis are used to realize the measurement and prediction of this index on the basis of the satisfaction investigation on the 27 high-speed railways in china. It is demonstrated that instead of only calculating the economic benefits brought by the construction of high-speed railway, this indicator can find the most urgent railway to be improved by directly evaluate the existing railway facilities from the perspective of passenger satisfaction investigation.
\end{abstract}

Keywords: Analysis location-routing problem; bi-level genetic algorithm; rural logistics; enterprise profit

\section{$1 \quad$ Introduction}

The high-speed railway provides people with convenience but requires substantial financial investment. It is one of the ultimate objectives of transport researchers and administrators to evaluate the economic effectiveness of the construction in this area. However, due to the economic growth is related to various factors such as the enhancement of education levels or rising consumption, it is difficult to distinguish the benefits brought by the improvement of railway services. So that, there is a strong need to develop a better indicator to measure and forecast the rewards from the efforts on the operation of high-speed railway.

The induced traffic represents the new traffic volume appeared from the improvement of road condition (Goodwin 1992). Many studies in the field of transportation frequently forecast this index with fixed coefficients by various methods (Barr 2000; Hymel, Small and Dender 2010) and treat it as a basis for road traffic capacity design (Graham, McCoy and Stephens 2014; Noland 2001; Zolnik 2016) or calculation of congestion probability after urban traffic improvement (Moscholios et al 2015; Duranton and Turner 2011). From the perspective of Transportation Economics, the induced traffic can be conceived as the increased demand caused by the decrease of generalized transportation cost, which reflecting the urgency of people to reduce the transportation costs (Oum, Waters and Yong 1992; Goodwin 1992). In other words, the induced traffic is mainly determined by the satisfaction degree of the existing transportation infrastructure (i.e. the inter-city railways) (Graham and Glaister 2004). Therefore, instead of calculated by the coefficient given in advance, passenger satisfaction survey will be an effective means to evaluate the induced traffic which can become a useful index for quantifying the effectiveness of high speed railway construction (Cervero and Hansen 2002).

In consideration of the induced traffic, this research has taken the high-speed railways opened in China as the research object to verify the validity of the index. Moreover, we have explored the formation mechanism of the indicator with the Canonical Correlation Analysis (CCA) for using it in the future high-speed railway construction planning. The remaining parts of this paper are organized as follows. Section 2 introduces the study area. Next, the Matrix Completion (MC) for the satisfaction 
investigation is used in Section 3. Thereafter, CCA is applied from the recovered data in Section 4. Finally, Section 5 provides the conclusions of this study and discusses future research issues.

\section{$2 \quad$ Study Area and Data Survey}

The high-speed railway in China is in rapid development since the Beijing-Tianjin inter-city railway line has been completed on August 1, 2008. As of the end of 2015, Chinese high-speed railway operating mileage reached 19 thousand $\mathrm{km}$, ranking first in the world. Moreover, as the Figure 1 shows, the proportion of high-speed railway is gradually increasing as years went by and will up to a stunning $20 \%$ in 2020, covering $80 \%$ of large Chinese cities according to the latest published "13th Five-Year Plan".

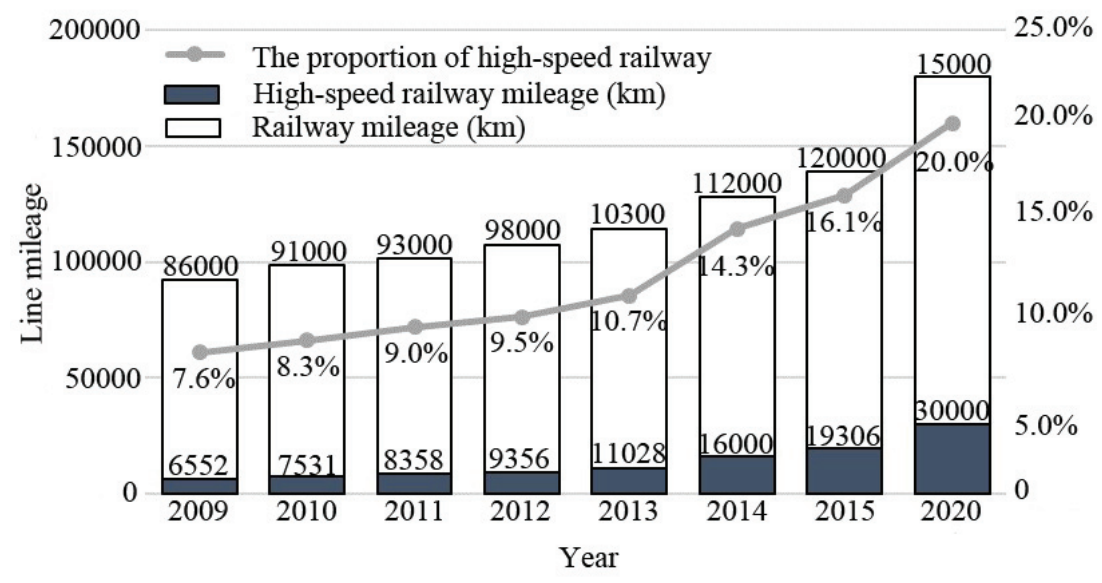

Figure 1. Development of high speed railway in China

The study area of this research is concentrated in the 27 intercity high-speed railways opened before the end of 2014 and the 40 cites they connected. On the bases of the influencing factors on passenger transport demand from economic geography and transportation economics (He and Zhao 2014; Ozuysal and Tanyel 2008; Wang and Shen 2003), we collect the data of the economic aggregate, population, similar degree of industrial structure and passenger satisfaction of these cities before the corresponding high-speed railways completed as the analytical variables. Meanwhile, the data of the economic growth of these cities and the induced traffic after the high-speed railways has opened one year are counted as the evaluation variables in Table1.

Table 1. Selection of variables

\begin{tabular}{cc}
\hline Analytical variables & Evaluation variables \\
\hline $\begin{array}{c}\text { Total population } \\
\text { Similar degree of industrial structure } \\
\text { Economic aggregate }\end{array}$ & Induced traffic \\
\hline $\begin{array}{c}\text { Distance } \\
\text { Passenger satisfaction }\end{array}$ & Economic growth \\
\hline
\end{tabular}

\section{$3 \quad$ Satisfaction Investigation}

Unlike the rest of data can be acquired by the statistical yearbook, passenger satisfaction with the railways can only be obtained by the investigation, so that a new research method from the recommendation system (Herlocker et al 2004; Hung 2005) is applied in this study to instead of the direct survey which required a huge volume of work.

In the process of satisfaction investigation, 3000 passengers giving a score from one to five depending on the satisfaction degree of their familiar existed railway services when the high-speed railways are 
uncompleted. Then we collect data to fill a matrix where the rows index each passenger and the columns index the railways but unfortunately, this matrix is only partially filled out with many missing answers because no one can familiar with all the 27 railways. In order to infer the missing data, many methods have been continually developed, including content based estimation (Deshpande and Karypis 2004; Melville, Mooney and Nagarajan 2002; Su et al. 2008), Collaborative Filtering (Hernando, Bobadilla and Ortega 2016; Kaleli 2014; Park et al. 2015), and MC (Candès and Recht 2009; Recht 2011). Under the assumption of low-rank matrix, MC can perfectly recover the lost information with a high probability when the singular value and the number of observed entries about this matrix satisfying certain conditions (Recht, Fazel and Parrilo 2010).

As the recommendation system suggested, the evaluation of people for objective things is influenced by limited number of factors rather than entirely random (Bodapati 2008; Hirayama, Hyvärinen and Ishii 2016). In other words, the satisfaction matrix is hypothesized to be low rank in this research. Therefore, the missing data can be restored through the MC that to find the approximate linear relationship of the observed entries in high dimensional space by minimize the nuclear norm of the matrix in equation 1.

$$
\begin{array}{cl} 
& \min \operatorname{rank}(X) \\
\text { s.t } \quad X_{i j}=M_{i j}, \forall(i, j \in \Omega)
\end{array}
$$

where $X \in \mathbb{R}^{m \times n}$ is the decision variable and $\operatorname{rank}(\mathrm{X})$ is equal to the rank of the matrix $\mathrm{X}, \Omega \in \mathbb{R}^{m \times n}$ is the set of locations corresponding to the observed entries in the data matrix $\mathrm{M}$.

In this study, the nuclear norm in equation 2 which means the sum of the singular values over the constraint set is minimized to obtain the approximate solution of the NP-hard optimized problem in equation 1 (Candes and Plan 2010).

$$
\begin{array}{ll} 
& \min \|X\|_{*} \\
\text { s.t } \quad & X_{i j}=M_{i j}, \forall(i, j \in \Omega)
\end{array}
$$

However, due to the inevitable errors from the survey data, there may not have a low-rank matrix satisfying the equality condition in equation 2 (Keshavan and Montanari 2010). Therefore, by carrying out relaxation on the constraint, a convex optimization is given by

$$
\begin{aligned}
& \min \mu\|X\|_{*}+\frac{1}{2}\left\|P_{\Omega}(X)-P_{\Omega}(M)\right\|_{F}^{2} \\
& \text { s.t. } X \geq 0 \\
& P_{\Omega}(X)_{i j}= \begin{cases}x_{i j}, & \text { if }(i, j) \in \Omega \\
0, & \text { else }\end{cases}
\end{aligned}
$$

where, $\mu$ is the external parameter, and $P_{\Omega}$ only mapping to the no-zero sparse matrix subspace of $\Omega$. As we will discuss below, equation 4 which is equivalent to the equation 3 can be optimized efficiently via alternating direction method of multipliers (ADMM) which have first proposed in 1975 (Glowinski and Marrocco 1975).

$$
\begin{aligned}
& \min _{X, Z} \mu\|Z\|_{*}+\frac{1}{2}\left\|P_{\Omega}(Z)-P_{\Omega}(M)\right\|_{F}^{2} \\
& \text { s.t. } \quad Z=X \\
& \quad X \geq 0
\end{aligned}
$$

where, $\mathrm{Z}$ is the introduced matrix separation variable. The Augmented Lagrangian function of equation 4 is given by

$$
\Gamma(X, Z, \Lambda)=\mu\|Z\|_{*}+\frac{1}{2}\left\|P_{\Omega}(Z)-P_{\Omega}(M)\right\|_{F}^{2}+\langle\Lambda, Z-X\rangle+\frac{\rho}{2}\|Z-X\|_{F}^{2}
$$

where, $\Lambda \in \mathbb{R}^{m \times n}$ is a Lagrange multiplier and $\rho>0$ is a penalty parameter. The ADDM of equation 4 is to minimize the function $\Gamma$ for variables $\mathrm{X}, \mathrm{Z}$ and $\wedge$ in turn. In other words, three sub-problems which are presented in equation 5 will be generated. 


$$
\begin{gathered}
X_{k+1}=\arg \min _{X \geq 0} \Gamma\left(X, Z_{k}, \Lambda_{k}\right), \\
Z_{k+1}=\arg \min \Gamma\left(X_{k+1}, Z, \Lambda_{k}\right), \\
\Lambda_{k+1}=\Lambda_{k}+\gamma \rho\left(X_{k+1}-Z_{k+1}\right),
\end{gathered}
$$

where, $\gamma \in(0,0.618)$. The terms of equation $5 \mathrm{a}$ can be rearranged to get the equation 6 which can be decomposed into two sub problems in equation 7 .

$$
\begin{aligned}
& \quad \min \frac{1}{2}\left\|P_{\Omega}(Z)-P_{\Omega}(M)\right\|_{F}^{2}+\frac{\rho}{2}\left\|X-\left(Z_{k}-\frac{1}{\rho} \Lambda_{k}\right)\right\|_{F}^{2} \\
& \quad \text { s.t } \quad X \geq 0 \\
& \min \frac{1}{2}\left\|P_{\Omega}(Z)-P_{\Omega}(M)\right\|_{F}^{2}+\frac{\rho}{2}\left\|P_{\Omega}(X)-P_{\Omega}\left(Z_{k}-\frac{1}{\rho} \Lambda_{k}\right)\right\|_{F}^{2} \\
& \text { s.t } \quad P_{\Omega}(X) \geq 0
\end{aligned}
$$

and

$$
\begin{aligned}
& \min \left\|P_{\hat{\Omega}}(X)-P_{\hat{\Omega}}\left(Z_{k}-\frac{1}{\rho} \Lambda_{k}\right)\right\|_{F}^{2} \\
& \text { s.t } \quad P_{\hat{\Omega}}(X) \geq 0
\end{aligned}
$$

where, $\widehat{\Omega}$ is the complementary set of $\Omega$. The solution of equation 5 a is obtained in equation 8 by solve the sub-problems in equation 7 .

$$
\begin{gathered}
\left(X_{k+1}\right)_{\Omega}=P_{+}\left(\frac{1}{\rho+1} P_{\Omega}\left(M+\rho E_{k}\right)\right), \\
\left(X_{k+1}\right)_{\hat{\Omega}}=P_{+}\left(P_{\hat{\Omega}}\left(E_{k}\right)\right), \\
P_{+}(X)_{i j}= \begin{cases}x_{i j}, & \text { if } x_{i j}>0 \\
0, & \text { else }\end{cases}
\end{gathered}
$$

where, $E_{k}=Z_{k}-\frac{1}{\rho} \Lambda_{k}$. Then, in order to solve the equation $5 \mathrm{~b}$, we will delete the constant term to convert it into a more compact form in equation 9 and prove the following theorem.

$$
Z_{k+1}=\arg \min \mu\|Z\|_{*}+\frac{\rho}{2}\left\|Z-\left(X_{k+1}+\Lambda_{k} / \rho\right)\right\|_{F}^{2}
$$

Theorem 1. Let $\mathrm{Y}$ be an $\mathrm{m} \times \mathrm{n}$ matrix of rank t, and take $Y=U_{Y} \operatorname{Diag}(\omega) V_{Y}^{T}$ as its singular value decomposition with $U_{Y} \in R^{m \times t}, \omega \in R_{+}^{t}, V_{Y} \in R^{n \times t}, v \geq 0$. If $S_{v}(\omega)=\varpi$ with which

$$
\varpi_{i}=\left\{\begin{array}{l}
\omega_{i}-v, \quad \text { if } \omega_{i}-v>0 \\
0, \quad \text { else }
\end{array}\right.
$$

then the $A=S_{v}(Y)=U_{Y} \operatorname{Diag}\left(S_{v}(\omega)\right) V_{Y}^{T}$ is an optimal solution to the following problem

$$
\min _{A \in R^{m \times n}} f(A):=v\|A\|_{*}+\frac{1}{2}\|A-Y\|_{F}^{2}
$$

Proof There is a unique minimum point of equation 10 because of the function $h_{0}(A)=v\|A\|_{*}+\|A-Y\|_{F}^{2} / 2$ is strictly convex. Thereafter, according to the definition of sub-gradient, 
the $\hat{A}$ is the minimum point of equation 10 if and only if zero is the sub-gradient of $h_{0}(A)$ at point $\hat{A}$ follows form

$$
0 \in \hat{A}-Y+v \partial\|\hat{A}\|_{*}
$$

Let $\hat{A}=S_{v}(Y)$, and we will prove it satisfying the condition in equation 11 . The expression of singular value decomposition of $\mathrm{Y}$ can also be given by

$$
Y=U_{0} \Sigma_{0} V_{0}^{T}+U_{1} \Sigma_{0} V_{1}^{T}
$$

where, $U_{0}$ and $V_{0}$ are the singular value vectors which have lager singular value than v; $U_{1}$ and $V_{1}$ corresponding to the residual singular value vectors which have smaller singular value than v. So that

$$
\begin{gathered}
\hat{A}=U_{0}\left(\Sigma_{0}-v I\right) V_{0}^{T} \\
Y-\hat{A}=v\left(U_{0} V_{0}^{T}+W\right) \\
W=v^{-1} U_{1} \Sigma_{1} V_{1}^{T}
\end{gathered}
$$

Moreover, $U_{0}^{T} W=0$ and $W V_{0}=0$ according to the orthogonal relationship between $U_{0}, V_{0}$, $U_{1}$, and $V_{1}$. On the other hand, because of each element of $\Sigma_{1}$ is equal or less than $v$, the $\|w\|_{2} \leq 1$. In summary, we can conclude that $Y-\widehat{A} \in \partial\|\widehat{A}\|_{*}$ and theorem 1 are proved. Hence, the solution of equation 9 is acquired based on theorem 1 follows form

$$
Z_{k+1}=S_{\frac{\mu}{\rho}}\left(X_{k+1}+\frac{1}{\rho} \Lambda_{k}\right)
$$

Finally, the ADDM which is applied in equation 4 will run as the following iteration.

$$
\begin{aligned}
& \left(X_{k+1}\right)_{\Omega}=P_{+}\left(\frac{1}{\rho+1} P_{\Omega}\left(M+\rho E_{k}\right)\right), \\
& \left(X_{k+1}\right)_{\hat{\Omega}}=P_{+}\left(P_{\hat{\Omega}}\left(E_{k}\right)\right), \\
& Z_{k+1}=S_{\frac{\mu}{\rho}}\left(X_{k+1}+\frac{1}{\rho} \Lambda_{k}\right), \\
& \Lambda_{k+1}=\Lambda_{k}+\gamma \rho\left(X_{k+1}-Z_{k+1}\right)
\end{aligned}
$$

\section{Canonical Correlation Analysis}

In this research, the two index of the induced traffic and the economic growth, which are not independent of each other will jointly, evaluated the feasibility of the high-speed railway construction. Therefore, the CCA which acts as a dimensionality reduction technology (Fujikoshi and Veitch 1979; Hwang et al 2013) is used in this study to show the global correlation between the analytical variables $X \in R^{p \times n}$ and the evaluation variables $Y \in R^{q \times n}$, where the sample set is $\left\{\left(x_{i}, \mathrm{y}_{i}\right)\right\}_{i=1}^{n} \in R^{p} \times R^{q}$.

The purpose of CCA is to find two linear combinations $w_{x} \in R^{p}$ and $w_{y} \in R^{p}$ for $X$ and $Y$ respectively (Chen, Liu and Carbonell 2012; Sun, Ji and Ye 2011), which can maximize the correlation between the random variables $x_{c a n}=w_{x}^{T} x$ and $y_{c a n}=w_{y}^{T} y$, where the $w_{x}$ and $w_{y}$ are called the correlation coefficients and the $x_{c a n}$ and $y_{c a n}$ are known as the canonical variables in CCA. This process can be specifically expressed as the maximization problem in equation (12),

$$
\rho=\frac{w_{x}^{T} C_{x y} w_{y}}{\sqrt{w_{x}^{T} C_{x x} w_{x} \cdot w_{y}^{T} C_{y y} w_{y}}}
$$

where, 
$\rho$ : Pearson correlation coefficient between canonical variables,

$C_{x x}$ : Covariance matrix within the $\mathrm{X}$,

$C_{y y}$ : Covariance matrix within the $\mathrm{Y}$,

$C_{x y}$ : Covariance matrix between the $\mathrm{X}$ and $\mathrm{Y}$, and $C_{x y}=C_{y x}^{T}$.

The method of solving this problem is to find a pair of suitable coefficients $w_{x}$ and $w_{y}$ which enable the denominator to be equal to 1 and maximize the numerator at the same time in equation 12 , which can be expressed as an optimization problem below

$$
\begin{aligned}
& \max _{w_{x}, w_{y}} w_{x}^{T} C_{x y} w_{y} \\
& \text { s.t } \quad w_{x}^{T} C_{x x} w_{x}=1, \quad w_{y}^{T} C_{y y} w_{y}=1
\end{aligned}
$$

The Lagrange multiplier method is used in this study to transform the equation 13 into a Lagrange function in equation 14

$$
L=w_{x}^{T} C_{x y} w_{y}-\frac{\lambda_{1}}{2}\left(w_{x}^{T} C_{x x} w_{x}-1\right)-\frac{\lambda_{2}}{2}\left(w_{y}^{T} C_{y y} w_{y}-1\right)
$$

Moreover, we can treat the optimization problem as solving the eigenvalue in equation 15 by writing the solution of equation 14 in matrix form.

$$
\left[\begin{array}{c}
X Y^{T} \\
Y X^{T}
\end{array}\right]\left(\begin{array}{l}
w_{x} \\
w_{y}
\end{array}\right)=\lambda\left[\begin{array}{c}
X X^{T} \\
Y Y^{T}
\end{array}\right]\left(\begin{array}{l}
w_{x} \\
w_{y}
\end{array}\right)
$$

Thereafter, let the eigenvalues $\lambda$ order as $\lambda_{1} \geq \lambda_{2} \cdots \geq \lambda_{r} \geq 0$ and correspond to the feature vector of nonzero eigenvalues $w_{x i}$ and $w_{y i}$, where $i=1, \cdots r$ and $r \leq \min (p, q)$. Then, we can obtain the Canonical correlation coefficient by inserting the $w_{x i}^{T} x$ and $w_{y i}^{T} y$ which come from the feature extraction of the corresponding pair of feature vector (i.e. correlation coefficients) $w_{x i}$ and $w_{y i}$ into equation 12 .

\subsection{Analysis result}

Table 2. Canonical correlation coefficient and canonical redundancy analysis

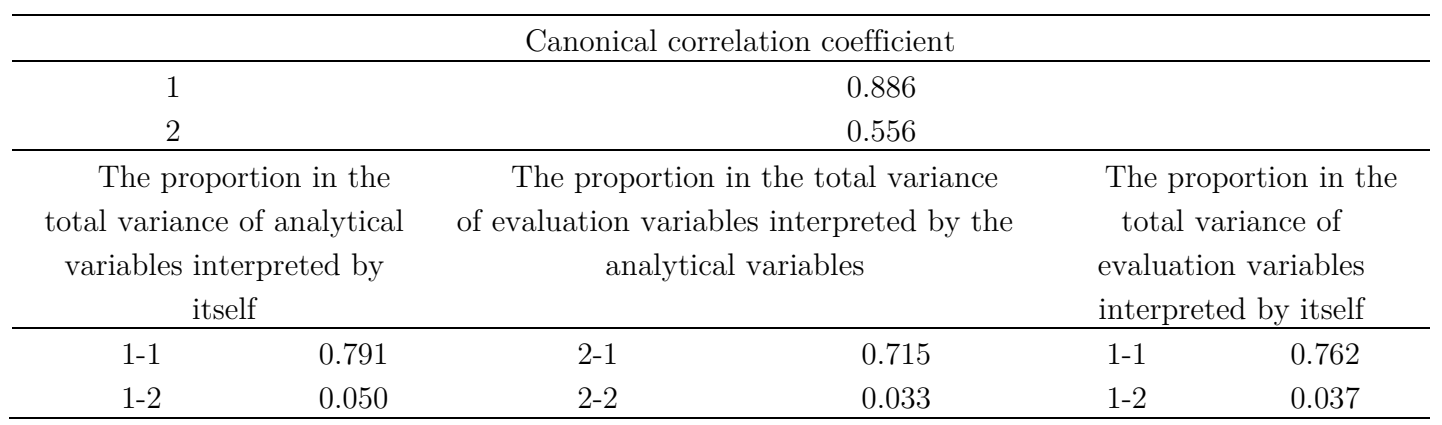

On the basis of data collected in section 3, the canonical correlation and redundancy analysis result of analytical variables and evaluation variables are shown in Table 2. The correlation of first canonical correlation coefficient 0.886 is stronger than the second canonical correlation coefficient 0.556 . Moreover, under the significant level of 0.05 , the test results show that only the first canonical correlation coefficient is significant which illustrate the strong correlation between the analytical variables and the evaluation variables.

Furthermore, the standardized coefficients of each variable for the first and second canonical correlation coefficient are shown in Table 3 which demonstrated that the passenger satisfaction with the largest coefficient in the analytical variables has a significant influence on the evaluation variables. 
Meanwhile, the coefficient of induced traffic is far larger than the economic growth illustrate that it has great representation on the utility evaluation of high speed railway construction.

Table 3. Canonical correlation coefficient and canonical redundancy analysis

\begin{tabular}{ccc}
\hline Analytical variables & \multicolumn{2}{c}{ Standardized coefficient } \\
\hline & 1 & 2 \\
\hline Total population & 0.213 & 0.293 \\
Similar degree of industrial structure & 0.324 & 0.633 \\
Average per capita GDP & 0.166 & 0.271 \\
Distance & -0.193 & -0.401 \\
Passenger satisfaction & 0.801 & 0.746 \\
\hline Evaluation variables & \multicolumn{2}{c}{ Standardized coefficient } \\
\hline Induced traffic & 0.526 & 0.385 \\
Economic growth & 0.113 & 0.206 \\
\hline
\end{tabular}

\subsection{Application}

Before the application of the model, we will validate the effectiveness of the evaluation index according to the actual opening sequence about the high-speed railways in section 2 . The index, which shows the utility of each high-speed railway operation, is calculated by equation 16 based on the data and the standardized coefficient in section 3 and 4 respectively.

$$
Z=\sum_{i} y_{i} b_{i}
$$

where,

$Z$ : Utility index matrix of the high-speed railway operation,

$b_{i}$ : Standardized coefficient for the first canonical correlation coefficient of the evaluation variable $i$ in section 4 ,

$y_{i}$ : Value of the evaluation variable $i$ in section 3

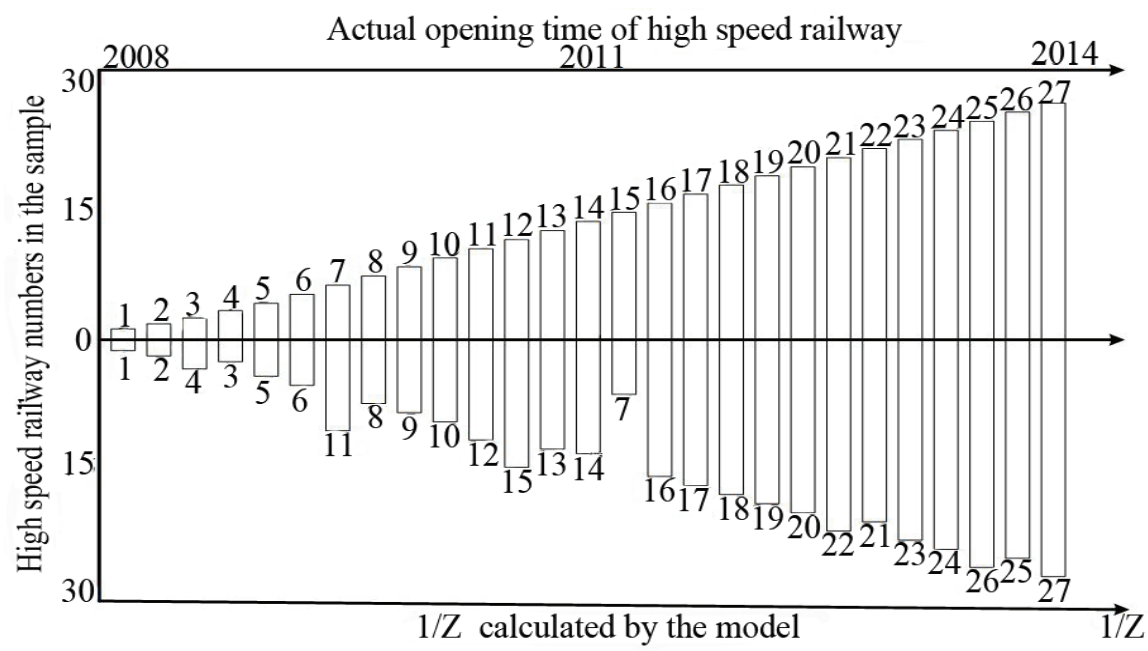

Figure 2. Opening sequence comparison of the high-speed railways

Then, as the figure 2 shows, all the railways are numbered as the actual opening sequence from 2008 to 2014 to make a contrast with the numbers rearranged by the size of $\mathrm{Z}$. It is apparently that the similarities of the two sequences respectively represented the opening sequence of these high-speed railways from the actual conditions and the model calculation reach to $65 \%$, which can be a strong evidence for the effectiveness of the evaluation index. 
The Kunming is the capital city of Yunnan province which is the last major city without the highspeed railway in china. In this research, the index is applied to help the policymakers decide which adjacent city should be the first to connect with Kunming by the high-speed railway in the actions below.

Phase-1: Collecting the data of Kunming and the adjacent cities from the statistical yearbook to calculate the analytical variables for each planed high-speed railway.

Phase-1: Carrying out the satisfaction investigation on the existing railways of the residents in these cities and recovering the missing data in satisfaction matrix by MC to calculate the average satisfaction of each railway as the analytical variable.

Phase-3: Predict the utility index by equation 17 for each planned high-speed railway and order them as size of $\mathrm{Z}$ in Figure 3.

$$
Z=\sqrt{\rho} \sum_{i} x_{i} a_{i}
$$

where,

$Z$ : Utility index matrix of the high-speed railway operation,

$\rho$ : The largest canonical correlation coefficient in section 4.1,

$a_{i}$ : Standardized coefficient for the first canonical correlation coefficient of the analytical variable $i$ in section 4 ,

$x_{i}$ : Value of the analytical variable $i$ in phase 1 and 2 ,

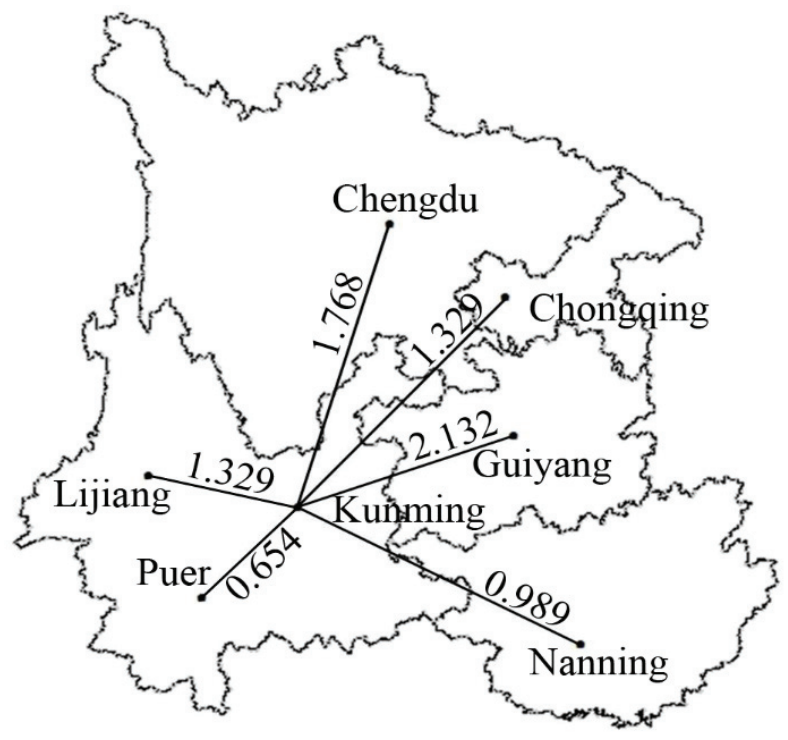

Figure 3. Utility evaluation of high speed railways connecting Kunming

As show in Figure 3, the high-speed railway which connects the Kumming and Guizhou has the largest $\mathrm{Z}$ which means more profit than the others when it has been built in this research, and the opening sequence of others should be ordered as Kuming-Chengdu, Kuming-Chongqing, Kuming-Lijiang, Kuming-Nanning, Kuming-Puer.

\section{Conclusions}

Considering the enormous scale of high-speed railway construction in China, the induced traffic is being added as a new perspective to evaluate the feasibility of the investment in this area. Through directly reflecting the urgency of improving the railway service in different areas, the induced traffic is more sensitive on the demand of efforts in the transportation than the economic index. Moreover, it is also found that after the problem of data acquisition difficulties is solved by MC, the degree of satisfaction with the existing railways plays the most important role in the emerging of the induced traffic. 
In this study, because of the huge workload, the data collection cannot be performed enough times, and we do not take into account the data of cities along the railways. In addition, the effect of each railway is assumed to be independent of each other in order to avoiding the network effect caused by the railway network. This situation should be improved in the future by finding more appropriate means to quantify this effect.

Acknowledgments. This research is supported by Program for New Century Excellent Talents in University (NCET-13-0655), National Natural Science Foundation of China $(71571011 ; 71390332)$ and Fundamental Research Funds for the Central Universities (2016JBM028).

\section{References}

1. Barr, L. Testing for the significance of induced highway travel demand in metropolitan areas. Transportation Research Record: Journal of the Transportation Research Board, 2000, 17(6), 1-8.

2. Bodapati, A. V. Recommendation systems with purchase data. Journal of marketing research, 2008, 45(1), 77-93.

3. Candes, E. J., \& Plan, Y. Matrix completion with noise. Proceedings of the IEEE, 2003, 98(6), 925-936.

4. Candès, E. J., \& Recht, B. Exact matrix completion via convex optimization. Foundations of Computational mathematics, 2010. 9(6), 717-772.

5. Cervero, R., \& Hansen, M. Induced travel demand and induced road investment: a simultaneous equation analysis. Journal of Transport Economics and Policy (JTEP), 2002, 36(3), 469-490.

6. Chen, X., Liu, H., \& Carbonell, J. G. Structured Sparse Canonical Correlation Analysis. In AISTATS (2012. Vol. 12, pp. 199-207).

7. Deshpande, M., \& Karypis, G.Item-based top-n recommendation algorithms. ACM Transactions on Information Systems (TOIS), 2004. 22(1), 143-177.

8. Duranton, G., \& Turner, M. A. The fundamental law of road congestion: Evidence from US cities. The American Economic Review, 2011, 101(6), 2616-2652.

9. Estimation of dimensionality in canonical correlation analysis. Biometrika, 1979, 66(2), 345-351.

10.Goodwin, P. B. A review of new demand elasticities with special reference to short and long run effects of price changes. Journal of transport economics and policy, 1992. 155-169.

11.Graham, D. J., \& Glaister, S. Road traffic demand elasticity estimates: a review. Transport reviews, 2004, 24(3), 261-274.

12.Glowinski, R., \& Marrocco, A. On the solution of a class of non-linear dirichlet problems by a penalty-duality method and finite elements of order one. In Optimization Techniques IFIP Technical Conference (1975. pp. 327$333)$.

13.Hung, L. P. A personalized recommendation system based on product taxonomy for one-to-one marketing online. Expert systems with applications, 2005, 29(2), 383-392.

14.Park, Y., Park, S., Jung, W., \& Lee, S. G. Reversed CF: A fast collaborative filtering algorithm using a k-nearest neighbor graph. Expert Systems with Applications, 2015. 42(8), 4022-4028.

15.Recht, B. A simpler approach to matrix completion. Journal of Machine Learning Research, 2011. 12(Dec), 34133430 .

16.Recht, B., Fazel, M., \& Parrilo, P. A. Guaranteed minimum-rank solutions of linear matrix equations via nuclear norm minimization. SIAM review, 2010. 52(3), 471-501.

17.Sun, L., Ji, S., \& Ye, J. Canonical correlation analysis for multilabel classification: A least-squares formulation, extensions, and analysis. IEEE Transactions on Pattern Analysis and Machine Intelligence, 2011, 33(1), 194-200.

18.Zolnik, E. J. Inducing Demand by Expanding Road Capacity: Controlling for the Rebound Effect. Annals of the American Association of Geographers, 2016, 1-16. 